Technical Section

\title{
Perceptual 3D rendering based on principles of analytical cubism
}

\author{
Sami Arpa*, Abdullah Bulbul, Tolga Capin, Bulent Ozguc \\ Bilkent University, Turkey
}

\section{A R T I C L E I N F O}

\section{Article history:}

Received 14 October 2011

Received in revised form

13 June 2012

Accepted 14 June 2012

Available online 28 June 2012

Keywords:

Cubism

Non-photorealistic rendering

Art

Computer graphics

\begin{abstract}
A B S T R A C T
Cubism, pioneered by Pablo Picasso and Georges Braque, was a breakthrough in art, influencing artists to abandon existing traditions. In this paper, we present a novel approach for cubist rendering of 3D synthetic environments. Rather than merely imitating cubist paintings, we apply the main principles of analytical cubism to 3D graphics rendering. In this respect, we develop a new cubist camera providing an extended view, and a perceptually based spatial imprecision technique that keeps the important regions of the scene within a certain area of the output. Additionally, several methods to provide a painterly style are applied. We demonstrate the effectiveness of our extending view method by comparing the visible face counts in the images rendered by the cubist camera model and the traditional perspective camera. Besides, we give an overall discussion of final results and apply user tests in which users compare our results very well with analytical cubist paintings but not synthetic cubist paintings.
\end{abstract}

(c) 2012 Elsevier Ltd. All rights reserved.

\section{Introduction}

Establishing a sense of realism in computer graphics has, until recently, been the main concern. With the realism goal nearly achieved, however, non-photorealistic and artistic rendering techniques [1-3] have started to garner more attention.

Cubism, pioneered by Pablo Picasso and Georges Braque, was a breakthrough in art, influencing artists to abandon existing traditions. It led to the emergence of modern art during a period of crisis that "the modern artist was heir to a tradition that had come to identify an object with its pictorial projection" [4]. In cubist paintings, we can perceive a multi-perspective projection of objects which creates ambiguity for overall composition. Differently than traditional one point perspective, artists show essential information of the content as much as possible by using multiple viewpoints. Cubism has its own evolution between 1906 and 1919. Although philosophy behind remains the same, its style has changed through these years. Two main periods of cubism are Analytical Cubism and Synthetic Cubism. Analytical Cubism covers the work of Picasso and Braque from 1908 until 1912 and mostly deals with the geometry of this new multi-view projection technique. On the other hand, during Synthetic Cubism period artists worked on new materials and combined them on canvas.

The philosophy and technique of cubism influenced not only artists, but also scholars and scientists from different disciplines. For example, various multi-perspective camera approaches have been introduced in the computer graphics field. Most of proposed

\footnotetext{
Article was recommended for publication by J. Collomosse

* Corresponding author. Tel.: +90 5447826508.

E-mail address: msamiarpa@gmail.com (S. Arpa).
}

methods provide a larger view of the scene than traditional perspective view using one camera or multiple camera models. Although radical spatial imprecision, clearly exhibited in all cubist paintings, has been addressed by several image based methods; for $3 \mathrm{D}$, a comprehensive model giving solution for both multiperspective view and spatial imprecision has not been proposed. In this paper, we describe a rendering method that uses principles of analytical cubism when generating images from synthetic 3D content (Fig. 1) by defining a flexible camera model ensuring expanded views with applied spatial imprecision. We also present a discussion of final outputs together with user evaluation results to validate the effectiveness of our approach.

The contributions of this paper are as follows:

- A cubist camera model to render synthetic 3D scenes. The proposed camera model enables multiple viewpoints with cubist-style faceting technique on a large and flexible camera surface. All viewpoints adjust their view angle (i.e. each facet adjusts its vieworientation) automatically to render important parts of the scene.

- A perceptually based spatial imprecision technique. Perceptually important parts of the 3D content are kept visible on the rendered image with this technique. The usage of perception techniques empowers artistic rendering approaches to bring artist's insight to the output.

- Several methods to provide a painterly effect. A border enhancement method, gradient mapping, and color transferring techniques are used to enhance artistic quality.

The paper is organized as follows: First, in Section 2, we discuss previous studies related to cubism, multi-perspective imaging, and artistic rendering. Then, we briefly explain cubism and its principles in Section 3, before giving the details of our approach in 

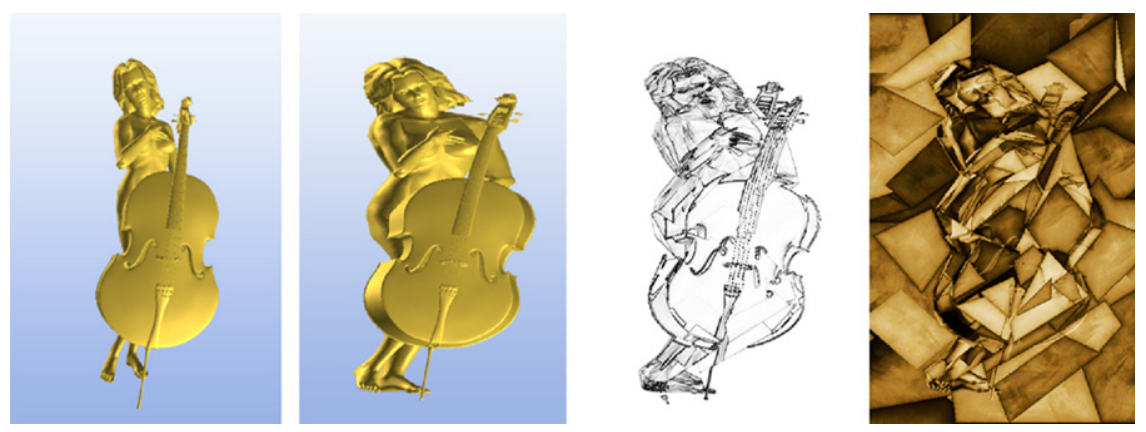

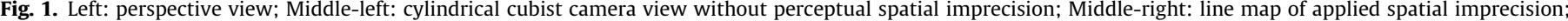
Right: final output of our result with applied perceptual spatial imprecision and artistic effects.

Section 4. Section 5 presents a detailed discussion of final outputs, and Section 6 concludes the paper.

\section{Related work}

Cubism as a movement breaking down traditional methods in art has inspired several works in computer graphics and imaging. Much of that has sought ways to introduce the principles used in cubist paintings, such as multi-perspective to computer rendering.

Multi-perspective rendering and non-linear projection. Inspired by cubism's multiple-viewpoints approach, several multi-perspective techniques have been proposed [5]. Most of these methods deal with a single camera combining multiple viewpoints. Glassner $[6,7]$ introduced an approach suited to ray tracing, in which rays are defined with NURBS surfaces. Löffelmann and Gröller [8] suggested an extended camera model that produces artistic effects by retaining the overall scene with ray tracing.

The general linear camera (GLC) model described by $\mathrm{Yu}$ and McMillan [9] generalizes linear cameras defined by a four dimensional ray space imposed by two planes, offering ray modeling flexibility. A camera model is constructed with three given rays, which allows implementing multi-perspective and non-pinhole camera models [10]. Another non-pinhole camera model, proposed by Popescu et al. [11], integrates several regions of interest in a 3D scene to render a single layer in a feed-forward fashion. Taguchi et al. [12] presented geometric modeling of multi-perspective images captured using axial-cone cameras. These approaches involve multi-perspective cameras with different viewpoints and ray groups.

A flexible projection framework with a single camera, proposed by Brosz et al. [13], can model nonlinear projections with parametric representation of the viewing volume.

The multi-perspective approach has also been widely used for designing algorithms for panoramas. Wood et al. [14] proposed a background panorama construction technique for the usage in traditional cel animations. Similarly, Rademacher and Bishop [15] presented a method to create a single image from multiple projection points.

More recently, interest has shifted to composite projections generated by the results of two or more cameras [13]. The main difficulty of composite projections is the occlusion of multiple projections from different view angles. Agrawala et al. [16] developed an interactive system attaching local cameras to a three dimensional space to generate multiprojection images of the scene by blending the results of the different angles. Likewise, Coleman and Singh [17] described a framework for the interactive authoring of projections obtained from linear perspective cameras.

A number of studies have addressed the multi-perspective approach in image space. Among these, Collomosse and Hall [18] and Agarwala et al. [19] proposed algorithms to combine the images rendered from different camera positions in various styles.
Cubism and artistic rendering. Apart from the works using cubist principles to develop new camera models, a number of studies aspire to render cubist-style paintings. In a prior work, Klein et al. [20] presented a method to create outputs evoking cubist and futurist paintings by using a space-time data cube from video. Along with using different view angles for the same content, their method also considers imprecision of object parts and a painterly style to enhance the similarity of their outputs to cubist paintings. Later, they generalized their methods to a set of NPR tools for video processing [21].

Collomosse and Hall [18] proposed a method to generate cubist-style outputs from images. As with video cubes, they use a series of images of the content as input to produce angular geometry in cubist art. The images are segmented with imagesaliency maps, and segments from different viewpoints are combined. The final composition is rendered with color and brush effects. However, Collomosse and Hall's work is image based and application of view-independent projection principle of cubism is dependent on the manually provided input images.

Influenced by the artistic styles of Kandinsky and Matisse, Song et al. [22] automatically produce highly abstract images using geometric shapes. A source image is segmented in different level of sizes and a variety of simple shapes are fitted to each segment. With a classifier, they automatically choose the segments which best represents the source image. The whole process creates an abstract form of the source image.

\section{Analytical cubism and properties to create cubist imagery}

In order to develop an accurate computational model representing analytical cubism and its rules, it is necessary to understand its concepts. To that end, we analyzed the works of Pablo Picasso and Georges Braque, given their pioneering role in Analytical Cubism. Although their paintings look like compositions of random shapes, the facets are ambiguous pieces of the content viewed from different angles, allowing a perspective that is not possible in a traditional projection. The main motivation behind cubist paintings is the desire to show that originality does not necessarily mean pictorial quality with a realistic perspective and unity [23]. Unconventional dimensions in the view and disharmony between object parts follow two major principles applied in cubist paintings:

- View-independent projection: In cubist paintings, radical discontinuities are emphasized through the manipulation of perspective, and artists exhibit a remarkable freedom from the point of view-dependency [24]. Instead of using a single viewpoint, multiple projections of a scene from different viewpoints are combined in a single projection. Thus, viewers can see more features of the content than in a linear perspective view. This multi-perspective approach has influenced 
research efforts in computer graphics, as presented in the previous section.

- Spatial imprecision: The radical approach that artists use to combine projections of independent viewpoints into one reveals this principle of cubism. Artists do not place importance on the continuity of projections in the final composite image, as in some of the multi-perspective rendering works mentioned in the previous section. Rather, they aim to keep all projections disjointed to some degree. This method creates extreme spatial imprecision in cubist paintings but does not cause the loss of object perception because key features of the subjects such as eyes and nose remain visible [25]. Different projections are painted into geometric shapes commonly in the style of quadrilaterals especially in the works of Picasso and Braque. In order to increase the effect of disharmony between different view projections, chiaroscuro - use of light and shadow - is also manipulated [24].

These two main principles do not specifically show how to create cubist imagery with specific rules. In surveying a range of cubist images, we derive a list of properties that are satisfied by existing artwork. These properties help to achieve view-independent projection and spatial imprecision.

Faceting: The dialectic between space and objects lead the evolution of cubism. Picasso and Braque developed the technique of faceting to create volumes and a tangible space on canvas. Faceting, which refers to creating different view facets of the space and content, is the core of analytical cubism and a very significant parameter to achieve both view-independent projection and spatial imprecision. While facets create a complex structure of planes, each of them represent an independent viewing volume going in different directions. In our proposed algorithm, we compare different faceting techniques for their similarity to existing cubist artwork. The following observations guide in determining the accurate faceting method:

- Facets help relating space and object. The degree of this relation changes in cubist paintings. Some artwork (Nude, Pablo Picasso, 1909-1910) have more legible relations, while some others (The Point of Ile de la Cité, Pablo Picasso, 1911) exhibit indistinguishable levels.

- The size of facets are smaller in salient parts of paintings. For instance, the facets forming face and clarinet in The Clarinet Player (Pablo Picasso, 1911) are smaller than other surrounding facets.

- Facets are commonly composed of vertical, horizontal and diagonal lines.

- Facet contours are bold and help viewers follow the form.

- The shapes of facets are not random, but are formed in relation with figures.

Ambiguity: Cubist paintings present as much essential information as possible, simultaneously visible, about the objects on the canvas, which is not possible with one-point traditional perspective [26]. The eye is not used to this kind of viewindependent projection. Hence, this process of re-creating visual reality causes ambiguity. While doing this, some unimportant parts of the object not giving any essential information are discarded. The amount of ambiguity depends on eccentricity of viewpoints. In the painting Portrait of Wilhelm Uhde (Pablo Picasso, 1910), viewpoints of facets are not so much disjointed which decreases ambiguity and makes the object more legible. On the other hand, The Portuguese (Georges Braque, 1911) exhibits a radical view-independency which creates total abstraction. As a matter of fact, the amount of ambiguity varies in cubist paintings. In our model, ambiguity is a variable, between $0^{\circ}$ and $360^{\circ}$, to determine wideness of the overall camera surface enabling to choose viewpoints on it for each facet. Increasing the value of ambiguity property gives a larger area of direction to choose viewpoints and a way to increase ambiguity of the whole composition.

Discontinuity: Discontinuity is another remarkable parameter for cubist paintings. Spatial imprecision is achieved by discontinuity between adjacent facets. On the other hand, the amount of discontinuity is not the same for all cubist paintings. For instance, adjacent facets in The Table (Georges Braque, 1910) form nearly a continuous structure, which makes the objects less ambiguous and more legible [26]. On the contrary, Violin and Palette (Georges Braque, 1909-1910) breaks the form by means of discontinuity. The level of discontinuity is represented with a variable in our model. Increasing discontinuity means increasing spatial imprecision for the overall composition. Practically, this variable limits the amount of view orientation for each facet from their initial direction. If discontinuity is chosen as zero, each facet keeps its initial direction, and in that way adjacent facets complete a continuous form.

In our approach, we propose algorithms to apply these cubist principles, using the properties discussed. First, our camera model allows covering a larger view than a traditional pictorial projection to establish a flexible ground for selection of multiple viewpoints and generation of ambiguity. Next, we offer a saliencybased spatial imprecision method to break up the unity of the composition into facets, which show essential information of the content, and create discontinuity.

\section{Cubist rendering approach}

\subsection{Overview}

We have developed our cubist rendering system according to the principles given in the previous section. Fig. 2 shows an overview of the proposed method. Our initial camera models provide a continuous expanded view of the content from a single camera position. In the second step, we apply a faceting algorithm to create different viewpoint areas and a perceptual imprecision technique to break up overall unity of the view. Finally, painterly

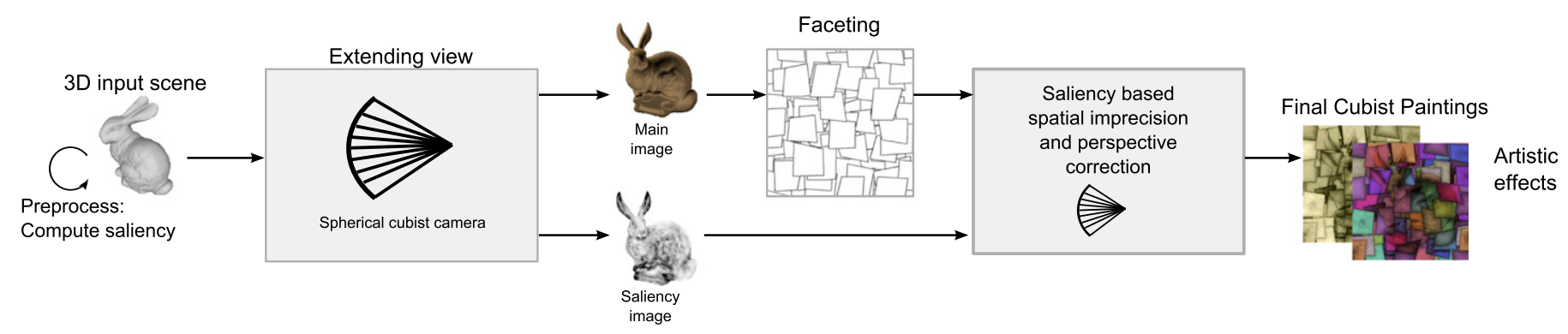

Fig. 2. Cubist rendering framework. 
effects are applied to manipulate chiaroscuro and enhance the image's artistic appeal.

\subsection{Extending view}

The first stage of our method applies the first major principle of cubism, view-independent projection, explained in Section 3. For this purpose, we use a multi-perspective projection method that focuses on a certain object space while showing the objects in that space in more detail. This projection method can be seen as the opposite of perspective projection, as rays converge at a focal point instead of spreading to the scene when applied to a ray tracing system. In this method, each ray has its own viewpoint conforming well with the view-independent projection principle.

We define two types of cubist cameras, both having the same underlying idea. The first camera type is the spherical cubist camera, where rays originate from a spherical surface and point to the center of the sphere (Fig. 3, left). This camera is capable of showing an object from all sides conforming to the view-independent projection principle of cubism. A spherical cubist camera can be defined by camera position $c_{p}$, camera size $s$, and convergence angles $\alpha_{x}$ and $\alpha_{y}$. If $\alpha_{x}$ and $\alpha_{y}$ are both $360^{\circ}$, the camera surrounds all the object. A ray at screen position $(x, y)$, where $x, y \in[-0.5,0.5]((0.5,0.5)$ being the top right corner), can be calculated for the spherical cubist camera as follows:

$M_{x}=$ rotation matrix on $\vec{u}$ axis by $x / \alpha_{x}$,

$M_{y}=$ rotation matrix on $\vec{h}$ axis by $y / \alpha_{y}$,
$\overrightarrow{\operatorname{rayDirection}(x, y)}=\left(M_{y} M_{x}(\overrightarrow{c d})^{T}\right)^{T}$,

$\overrightarrow{\text { rayOrigin }(x, y)}=\overrightarrow{c_{p}}-s \times \overrightarrow{\text { rayDirection, }}$

where $\vec{u}$ and $\vec{h}$ are the up and horizontal directions of the camera, and $\overrightarrow{c_{d}}$ is the camera direction. The convergence angle of the cubist camera has a significant role on the ambiguity of the resulting output. A higher convergence angle results in a more ambiguous rendering result, therefore the convergence angle is used as the ambiguity variable in our model.

Several 3D models, such as a standing human model, have a vertical elongated shape. A cylindrical camera surface could surround this type of models better than a spherical one. Thus, we define a second type of camera, cylindrical cubist camera (Fig. 3, right). The rays originating from the cylindrical camera surface converge to a vertical line instead of a single point as opposed to the spherical camera. Both of these camera types have advantages over each other: the spherical camera surrounds the scene both horizontally and vertically, while the cylindrical camera extends the view plane only in horizontal axis. According to the scene, either one of those camera types could be chosen. Both these camera models keep the linearity of the rays with non-linear camera surfaces, and this causes non-linear warps on the output.

To evaluate how these camera models depict a scene in more detail, we compared them against a regular perspective camera by counting the number of visible faces for each of the projection types. A large number of visible faces means that a large portion of the scene details is visible in the rendered image. Table 1 shows the result of this comparison. To compare the camera models
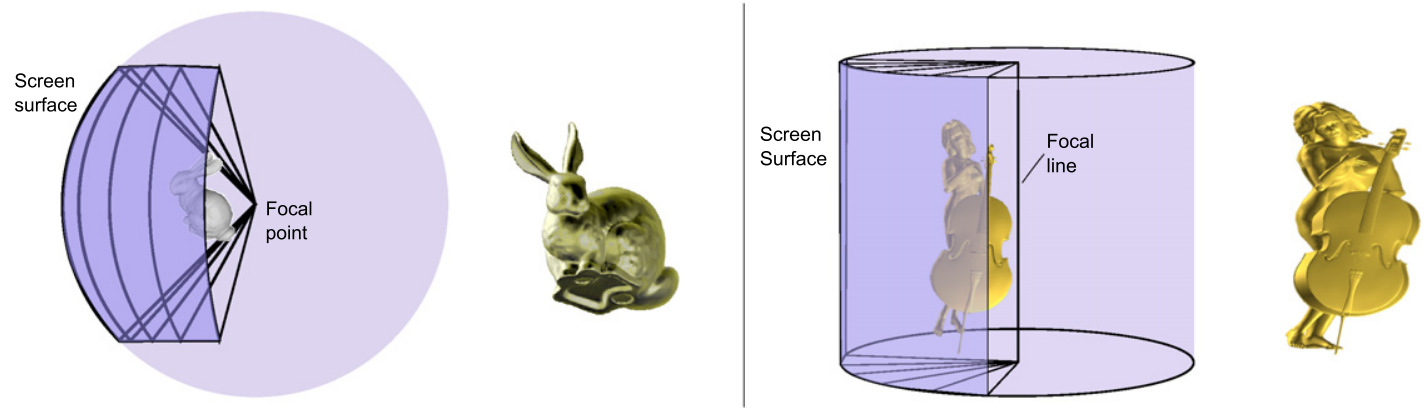

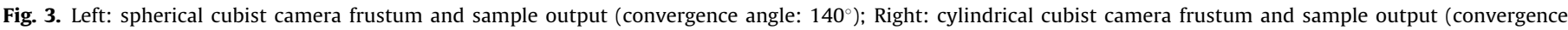
angle: $140^{\circ}$ ).

Table 1

Comparison of the camera models. (Angle denotes the convergence angle for the cubist camera and field of view for perspective cameras. In the upper figure sc stands spherical cubist camera.)

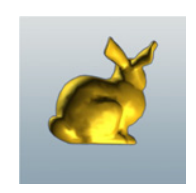

ort

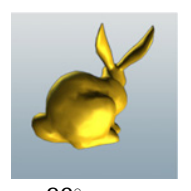

per90

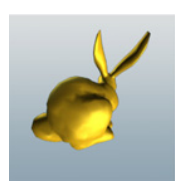

per120

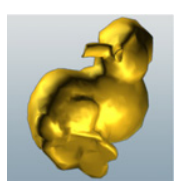

sc120

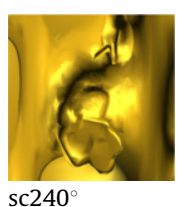

sc 240

Camera type angle Number of visible faces

\begin{tabular}{|c|c|c|c|c|c|c|c|}
\hline & & \\
\hline & & \multicolumn{2}{|c|}{ Sphere (1280F) } & \multicolumn{2}{|c|}{ Bunny (2K) } & \multicolumn{2}{|c|}{ Venus $(11 \mathrm{~K})$} \\
\hline \multirow[t]{3}{*}{ Spherical cubist } & $120^{\circ}$ & 1162 & $90.8 \%$ & 1325 & $66.3 \%$ & 5099 & $46.6 \%$ \\
\hline & $240^{\circ}$ & 1280 & $100 \%$ & 1629 & $81.5 \%$ & 7005 & $64.0 \%$ \\
\hline & $360^{\circ}$ & 1280 & $100 \%$ & 1745 & $87.3 \%$ & 7873 & $72.0 \%$ \\
\hline \multirow[t]{3}{*}{ Perspective } & $60^{\circ}$ & 378 & $29.5 \%$ & 717 & $35.9 \%$ & 4016 & $36.7 \%$ \\
\hline & $90^{\circ}$ & 245 & $19.1 \%$ & 653 & $32.7 \%$ & 3876 & $35.4 \%$ \\
\hline & $120^{\circ}$ & 121 & $9.5 \%$ & 576 & $28.8 \%$ & 3563 & $32.6 \%$ \\
\hline Orthogonal & $\mathrm{N} / \mathrm{A}$ & 840 & $50 \%$ & 840 & $42.0 \%$ & 4265 & $39.0 \%$ \\
\hline
\end{tabular}



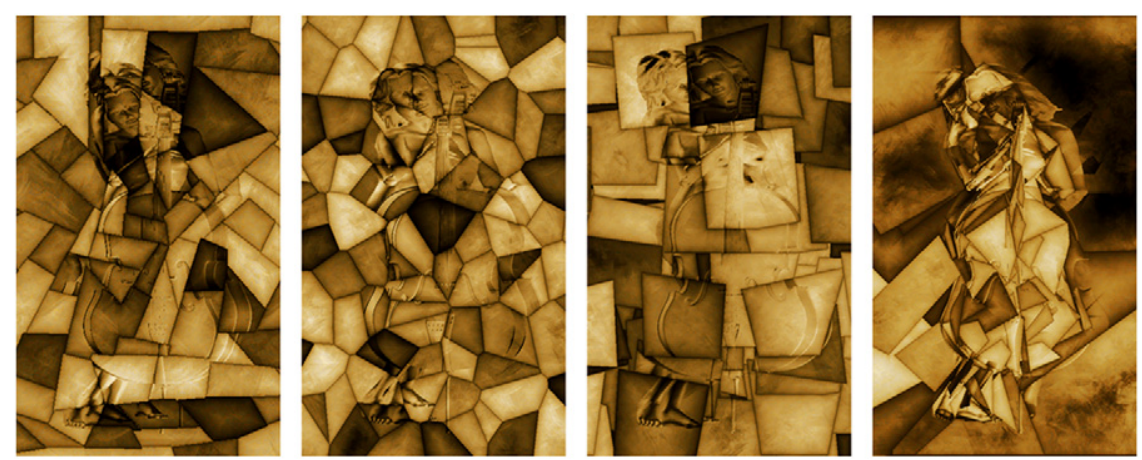

Fig. 4. Left: Constant; Middle-left: Voronoi; Middle-right: Patch; Right: Segment.

with 3D models of different natures, we used a simple sphere model, the Stanford Bunny, for a model of average detail, as well as a more detailed Venus model. The figures at the top of the table show the rendering results for the Stanford Bunny with each type of experimented cases. As shown in this table, the cubist camera is capable of showing notably more faces than the perspective and orthographic cameras. A user study for the perception of the cubist camera's ambiguity is given in Appendix A.

\subsection{Perceptual spatial imprecision}

As evident from the results in the previous section, our cubist camera enables view-independency to a great extent. The flawless continuity and homogeneity also exhibited by this camera are not associated with analytical cubist paintings, however, it lays the groundwork for choosing multiple viewpoints showing essential information of the objects. In this section, we introduce methods for breaking up the overall unity of this camera surface into view facets and achieving discontinuity by using a perceptual imprecision method.

\subsubsection{Faceting}

As discussed in Section 3, faceting is the most characteristic style of analytical cubism. Facets represent different viewpoints and engage space with the figures, which creates a tangible composition as a whole. We compare four different faceting techniques to segment the main view generated with the cubist camera into multiple viewpoints. The rules on faceting indicated in Section 3 guide to determine the most effective method among them.

The first strategy (Constant) uses constant filters to create facets. We have created such filters by extracting facet contours from sample analytical cubist paintings. In Fig. 4 left, Le Guitariste (Pablo Picasso) is used to create the filter image. Although this strategy is very convenient with respect to shape similarities to cubist paintings, it has the problems of scalability and flexibility. Hence, we present other algorithms for creation of filters dynamically for any scale.

The second strategy (Voronoi) uses Voronoi diagrams to create facets dynamically (Fig. 4, middle-left) similar to prior work on image-based cubist rendering methods [18]. The method accepts a number of facets $k$ as input and randomly chooses $k$ points on the empty filter image, which is created with the same resolution as the output image. To prevent regional accumulations of points, we use a grid system. For a grid with $m$ cells, random $k / m$ points are chosen for each cell. Thereafter, each pixel on the image is assigned to the point that has the shortest Euclidean distance from it.

The third strategy (Patch) proposes a method with the advantages of dynamic facet generation and similarity to the analytical structure of cubist paintings (Fig. 4, middle-right). In this strategy, a number of convex quadrilaterals possibly occluding each other
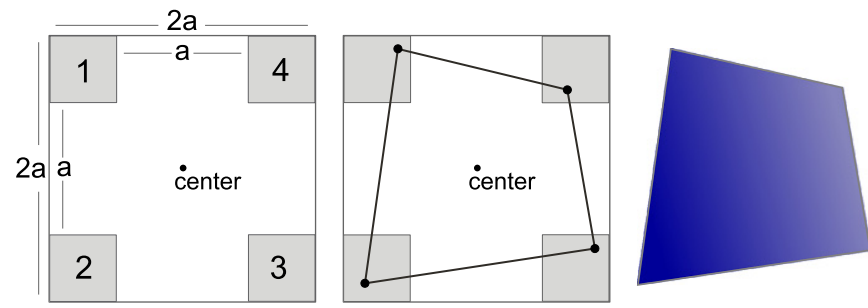

Fig. 5. Left: one point from each numbered area is randomly selected; Middle: the selected points are connected to form a quadrilateral; Right: the resulting facet.

are painted on the filter image. The number of facets $\left(n_{p}\right)$ and parameter $a$ defining approximate edge length of a facet are given for this strategy. Edge lengths change between $a$ and $2 a$. Fig. 5 shows a single facet generation. Increasing $n_{p}$ or $a$ results in facets that occlude each other more. To control this occlusion rate, we define another parameter $\left(r_{\text {occ }}\right)$ which could be used to define $a$ value indirectly. $r_{\text {occ }}$ determines the approximate rate of the total occluded area of the facets to the total visible area in the resulting image. When this parameter is used $a$ is calculated as follows:

$a=\sqrt{\frac{2 w \times h \times r_{o c c}}{5 n_{p}}}$,

where $w$ and $h$ stand for the width and height of the final image. We empirically select $n_{p}$ as 80 and $r_{o c c}$ as 3 in most cases. The constant $\frac{2}{5}$ is for compensating the difference between the expected area of a random facet and the smallest possible facet. All facets are generated in succession. To avoid empty regions in the filter image, center points, shown in Fig. 7, are selected randomly from the pixels that have not yet been assigned to facet.

Creating facets regardless of the structure of objects is the main problem of these three techniques. Nevertheless, it is very clear that facets help the eye to follow the form and they are not independent from the content in cubist paintings [26]. Our final technique (Segment) enables creating facets in relation with the objects in space (Fig. 4, right). To achieve this, we applied a similar segmentation procedure described by Song et al. [22]. The technique includes the following operations:

1. Segmentation: By using multiscale normalized algorithm by Cour et al. [27] we segment pre-rendered output (Fig. 6, a) in three different levels of detail. Large (Level 1, Fig. 6, c), medium (Level 2, Fig. 6, d) and small (Level 3, Fig. 6, e) size segments are obtained with different values of parameter $N$, which is a parameter to indicate the number of cuts. Each segment of each level is a candidate to be a facet for the final filter image.

2. Segment selection: The decision of selecting a segment for the final filter image is determined based on the average saliency 

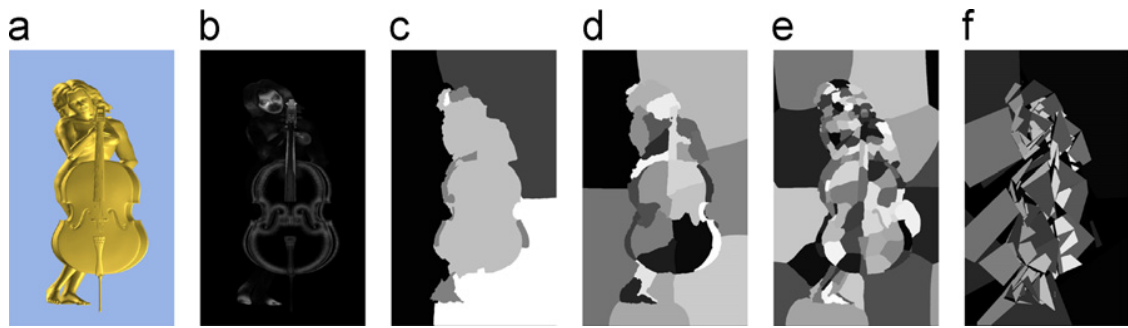

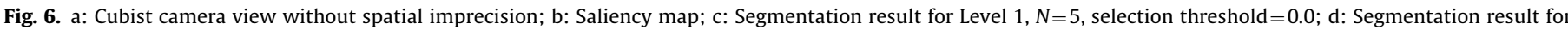
Level 2, $N=30$, selection threshold $=0.05$; e: Segmentation result for Level 3, $N=120$, selection threshold $=0.08$; f: Final filter.

value of each segment. Saliency shows visually significant areas of the content. Refer to Section 4.3.2 for detailed explanation of saliency calculation. By using the saliency map of pre-rendered content (Fig. 6, b), which includes saliency value for each pixel, average saliency value for each segment is calculated. Besides, we determine a selection threshold for each level. If the saliency value of a segment is larger than a selection threshold, it is chosen as a facet. The value of this threshold is the highest for Level 3 and smallest for Level 1. In this way, smaller segments have lower chance to be chosen as a facet. This decision is related with facet distribution in cubist paintings: smaller facets are included in high detail and contain important parts of the objects. Therefore, we select smaller facets for only highly salient parts, which are assumed as visually significant.

3. Overlapping facets and ordering: We also use the average saliency value for decision of occluding facets, and order them. The facet with the highest saliency value is selected as the frontier facet, since it tends to show more essential information.

4. Shape fitting: Lastly a shape of quadrilateral is calculated for each facet as its final form. Instead of using a complex shape fitting algorithm, we used a simple method to define segments boldly and keep ambiguity to a level. Left-most, right-most, upper-most and bottom-most pixels of the segment are determined as vertices of its quadrilateral for each segment (Fig. 6, f).

Our final strategy significantly satisfies most of the cubism rules discussed in Section 3. We also verify this issue by comparing the proposed four techniques with a user study, which is explained in Appendix A.

\subsubsection{Spatial imprecision using mesh saliency}

The extreme spatial imprecision applied to cubist paintings does not cause absolute loss of object perception. In most of Picasso and Braque's paintings, the subject, in some of which is a person and a musical instrument, is still perceivable, although it does not abruptly stimulate our visual perception since it has been divided into pieces morphologically resulting from multiple viewpoints and creates ambiguity. Nevertheless, because key features such as eyes, guitar strings, and noses are preserved, we perceive the content fairly quickly.

Rendering of these particular features of other parts notwithstanding creates the problem of selection. Indeed, in cognitive science, searching for the significant attributes of objects that captures our attention is essential work [28]. Thus, our method makes use of mesh saliency, proposed by Lee et al. [29], which is a perceptual approach to determine salient parts of a 3D object. Mesh saliency is based on the center-surround mechanism of the human visual system, which is basically related to the attentive interest on central regions that are different from their surroundings. In 3D, this mechanism is employed by calculating the difference of mean-curvature properties in the central and surrounding regions to determine the salient parts of 3D mesh
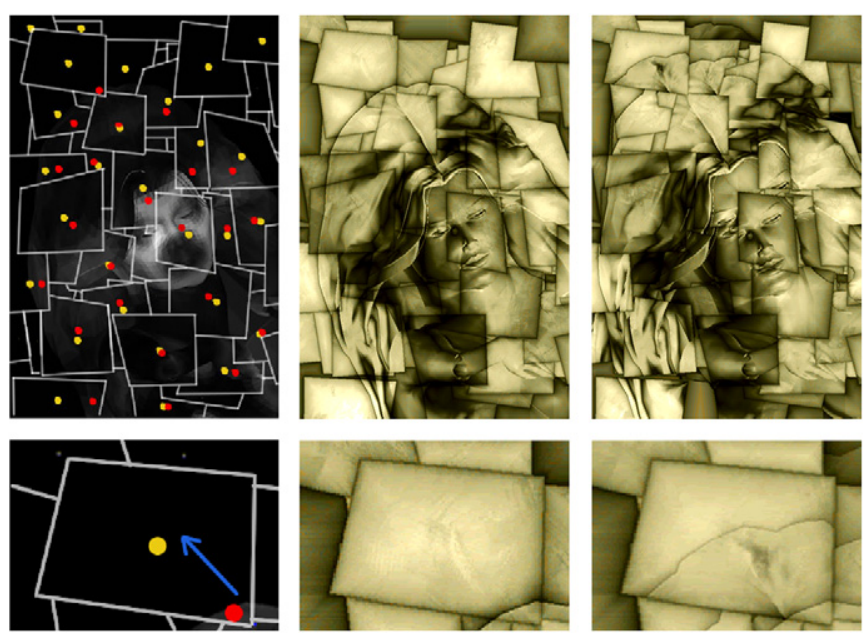

Fig. 7. Left-top: saliency map along with the calculated facet and saliency centers Lighter pixels indicate more salient parts. Yellow dots are facet centers and red dots are saliency centers; Middle-top: the result without spatial imprecision applied; Right-top: the result with spatial imprecision applied; Bottom: shift of view from facet center to saliency center for a specific facet. (For interpretation of the references to color in this figure caption, the reader is referred to the web version of this article.)

models. We refer the readers to the study of Lee et al. [29] for the details of this saliency computation method. Although this method does not consider the semantic properties of objects such as nose and eyes, these important regions could be identified by this model since they have significantly different geometric properties compared to their surroundings, as seen in Fig. 7, left.

After saliency values for each input model are calculated, our renderer decides which rays to cast for each pixel by considering the saliency orientation of each facet. Our facet-specific spatial imprecision technique includes the following sequence of operations (Fig. 7):

1. Construct the saliency map: This map is generated by a ray casting-based rendering operation, in which vertex saliencies are used as the material attributes. Each pixel of the saliency map (Fig. 7, left) is calculated by casting the rays for this pixel and computing the average saliency values of the intersected faces.

2. Calculate facet and saliency centers: All rays belonging to a facet must undergo the same operation to accomplish regional shifting. Hence, a facet center $(f c)$ and a saliency center $(s c)$ are calculated for each facet to determine the amount of shifting (Fig. 7, left). The facet center is the geometric center of the facet. As facets are generated and a facet id for each pixel is assigned with the operations indicated in the previous section, 
facet centers are calculated with the following formula:

$f c=\frac{\sum_{f \in F} f_{\text {pos }}}{|F|}$,

where $F$ is the set of all pixels in a facet and $f_{\text {pos }}$ is the position of pixel $f$. Similarly, saliency centers for each facet are calculated as follows:

$s c=\frac{\sum_{f \in F} f_{\text {pos }} f_{s l c}}{|F|}$,

where, $f_{\text {slc }}$ denotes the saliency of pixel $f$. Note that, set $F$ could also be extended to cover the neighboring pixels outside the facet, so that, in addition to the interior of a facet, exterior salient parts close to this facet could be considered while calculating the saliency center. The additional pixels to consider could be adjusted with a threshold indicating the neighborhood size. Increasing this threshold decreases the continuity among facets in the final image by enabling the saliency centers to be further away from the facet centers. Additionally, a facet orientation threshold could be used to limit the maximum distance between $f c$ and $s c$ to avoid extreme levels of re-orientation. If the distance between $f c$ and $s c$ exceeds this threshold $s c$ is repositioned such that its distance to $f c$ becomes the specified threshold. A smaller facet orientation threshold results in output images with less discontinuity. Facet orientation threshold is referred as discontinuity parameter to control the level of discontinuity between adjacent facets as explained in Section 5 in detail.

3. Orient the view from facet center to saliency center: In this step, rays belonging to a facet are re-oriented such that the facet shows the most salient parts at the center. Additionally, this step enables perspective view while keeping the rendered size of the content inside the facet (see Fig. 8).

Initially, the ray originating from the facet center $\left(r_{f c}\right)$ is redirected to the point that the ray originating from the saliency center $\left(r_{s c}\right)$ intersects with the 3D scene. Then, the new focal point becomes the value of the modified ray at focal distance and all rays belonging to the facet are redirected to this new focal point as shown in Fig. 8, middle.

This modification is sufficient for the facet to show the salient parts at the center; besides, further operations are necessary for a perspective view. Let $d_{a i}$ be the average intersection distance of the rays in this facet (excluding the rays that do not intersect). Rotating rays around their values at distance $d_{a i}$ enables keeping the rendered size of the shown region. Each ray is modified as follows:

$\overrightarrow{F(x, y)}=\overrightarrow{O(x, y)}+\overrightarrow{D(x, y)} \times d_{a i}$,
$\overrightarrow{O^{\prime}(x, y)}=\frac{\overrightarrow{O^{\prime}(x, y)} \times\left(d_{f}-d_{a i}-\alpha\right)+\overrightarrow{O(p c)} \times\left(d_{a i}+\alpha\right)}{d_{f}}$,

$\overrightarrow{D^{\prime}(x, y)}=\overrightarrow{F(x, y)}-\overrightarrow{O^{\prime}(x, y)}$,

where $d_{f}$ is the focal distance, $F(x, y)$ stands for the fixed point of rotation for the ray of pixel $(x, y)$, and $O(x, y)$ and $D(x, y)$ stand for the origin and direction of this ray respectively. $O^{\prime}(x, y)$ and $D^{\prime}(x, y)$ denote the modified (new) values of ray origin and direction. Here $\alpha$ is a term to control the perspective effect. If $\alpha$ is 0 , then the facet is rendered orthographically and increasing alpha increases the perspective. To select alpha according to a given field of view (FoV) angle, the following formula could be used:

$\alpha=\frac{d_{f} \times d_{a i} \times \tan \left(F o V \frac{|(x, y)-p c|}{y_{\max }-y_{\min }}\right)}{|O(x, y)-O(p c)|}$,

where $y_{\max }$ and $y_{\min }$ are the maximum and minimum $y$ values of the whole image (not only the facet). Note that the field of view for a facet is significantly small compared to the whole image, and the perspective and orthographics views do not differ considerably. Thus, alpha could be taken as zero for simplicity.

\subsection{Painterly effects}

Besides extending the view and spatial imprecision, our system also provides a process of basic painterly effects to apply the style of some cubist paintings in terms of colors and strokes.

Picasso and Braque's style was to boldly define facet borders. Our filter image is composed of pixels, each of which keeps only a facet id to define its owner facet. We apply a pixel neighborhood operation to detect facet edges and enhance them. For a pixel in the output image, its proximity to an edge is calculated by checking its neighborhood circle, shown in Fig. 9. If any pixel on
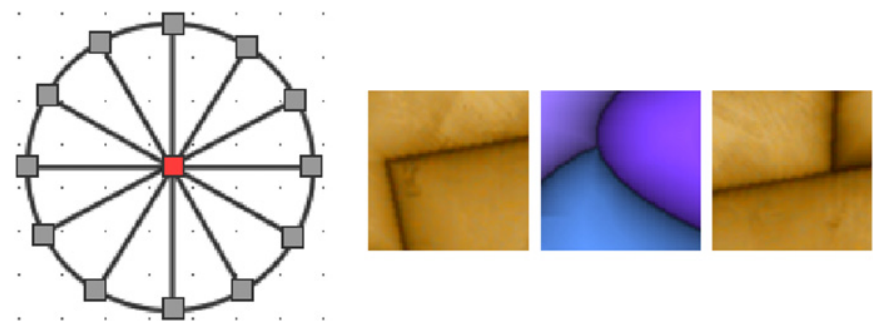

Fig. 9. Left: neighborhood circle for a given pixel; Right: sample border enhancements.
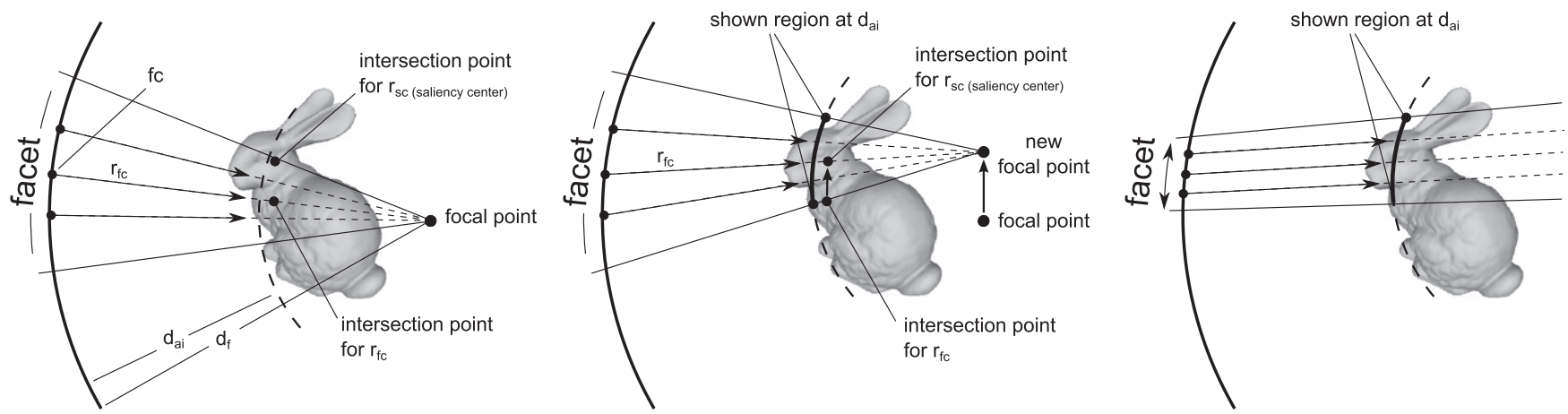

Fig. 8. Left: initial state of the rays in a facet; Middle: rays are re-oriented towards the salient area; Right: rays are modified for perspective view. 
the circle has a different facet id than the originating pixel, the pixels lying on the line between that pixel and the origin are checked until facet id is the same with the origin. In this manner, the closest distance to an edge is found and used for darkening the pixels of the output image with a polynomial interpolation as they get closer to a facet edge.

$p_{\text {color }}=p_{\text {color }} * \frac{m d+3 \sqrt{p_{d} m d}}{4 m d}, p_{d} \in|0, m d|$,

where $p_{\text {color }}$ stands for RGB color value for pixel $p, p_{d}$ stands for smallest distance of $p$ from a facet border and $m d$ defines the maximum distance for the effect.

A similar effect for enhancing facet borders is used for enhancing foreground-background discrimination. In this case, instead of proximity to facet borders, the proximity of background pixels to the foreground are calculated and used to alter the color of the background pixels such that object silhouettes become more visible.

By conceiving Picasso and Braque's color palettes, we also applied a simple color quantization. Picasso and Braque commonly used a limited number of colours; fine details were composed through a difference in luminance rather than hue. We use a painting from each artist as a reference image for color transfer. The pixels of the initially rendered output and the given cubist painting are sorted according to luminance values. Corresponding color value for each pixel is found with one-to-one linear mapping. Fig. 13 shows sample outputs with Le Guitariste
(Pablo Picasso, 1910) and Fig. 14, middle-left contains a result using Woman with a Guitar (Georges Braque, 1913).

Another feature of Picasso and Braque's paintings is the gradient overlays appearing on the corners or edges of some facets. Hence, we also create a gradient map for the corner of randomly chosen facets during rendering and apply this gradient map to the output. Fig. 10 shows several sample gradient mapping results.

\section{Results and discussion}

Measuring the quality of cubist outputs is not easy, even for art specialists, since cubism is an art movement which is evaluated as a disruption to excessive usage of technique for pictorial quality in art. Furthermore, as an NPR method, cubist rendering is challenging to evaluate objectively-Hertzmann [30] argues that experiments provide evidence but not proof that the NPR method works. Cubism comes forward with its philosophy of multi-perspective rendering and radical discontinuities rather than a specific pictorial style. Therefore the main focus of this work has been the proper application of principles and properties of analytical cubism, rather than imitating cubist paintings. In this direction, we present a comparison of our results (Figs. 12-14) with real cubist paintings, and the opinions of art critics about final results. We also performed five user studies (Appendix A) to observe the responses
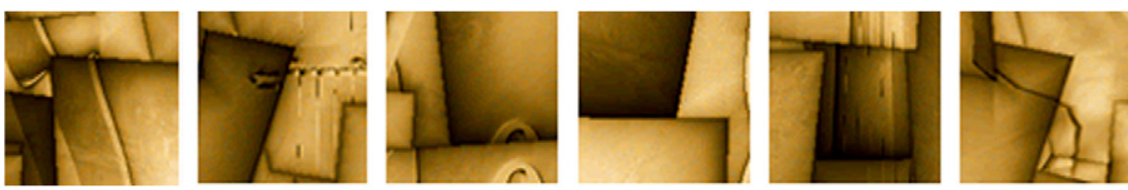

Fig. 10. Gradient mapping.
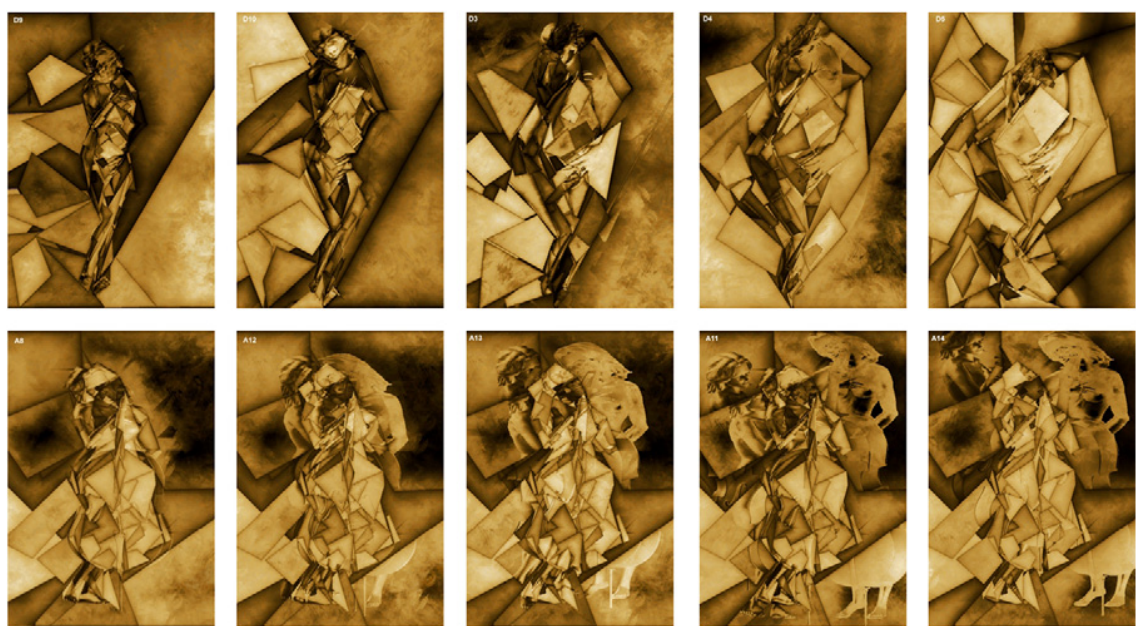

Fig. 11. Top: ambiguity is increased from left to right. Bottom: discontinuity is increased from left to right
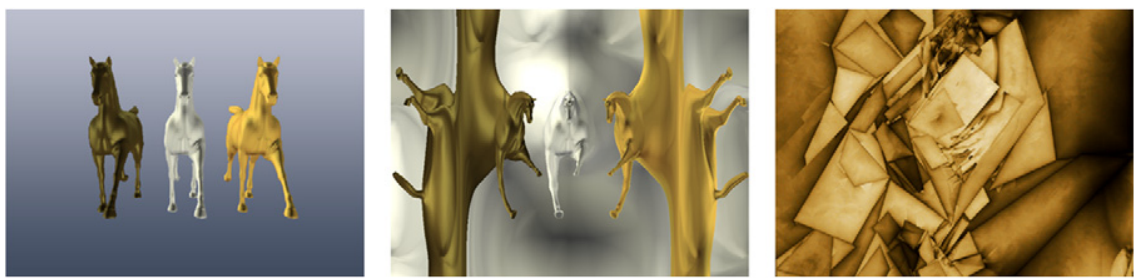

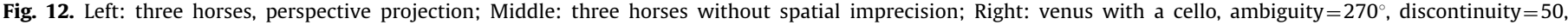
faceting = Segment. 
of viewers in comparison with cubist properties and real analytical cubist paintings, to guide our discussion.

\subsection{Ambiguity and discontinuity parameters}

Our current system suggests several interesting uses with ambiguity and discontinuity parameters. As mentioned earlier, the ambiguity parameter controls the convergence angle of the camera surface and the discontinuity parameter limits the saliency based orientation of each facet. Changing these two parameters varies the amount of ambiguity and discontinuity exhibited on the outputs.

Modifying the ambiguity in the cubist outputs change the legibility of the content and its relation with the space. As indicated in Section 3, some of cubist paintings such as Ma Joile (Pablo Picasso, 1912) have more ambiguous forms than others like Portrait of Wilhelm Uhde. When the two paintings are compared, it can be inferred that Ma Joile shows more information of the content by increasing the eccentricity of the viewpoints from the center. On the other hand, the content in Ma Joile is less legible since manipulation of traditional perspective is extreme. The ambiguity parameter in our system works with a similar idea. Increasing the ambiguity value causes the increase of convergence angle and the amount of eccentricity of viewpoints from the center (Fig. 11).

Similarly, the amount of discontinuity between adjacent facets vary in cubist paintings. When Braque's well known painting Violin and Palette is compared with another cubist painting The Table, there is significant difference in continuity of facets. Our saliency based spatial imprecision method enables creating discontinuity of adjacent facets. In order to obtain a variety of discontinuity results as in cubist paintings, the amount of discontinuity is controlled with a discontinuity parameter. This parameter limits view orientation freedom within each facet,
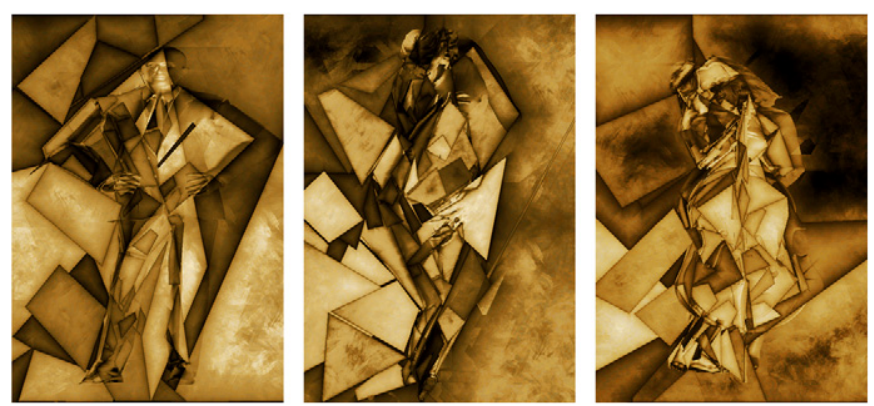

Fig. 13. Left: man, ambiguity $=120^{\circ}$, discontinuity $=0$, faceting $=$ Segment; Middle: venus with a cello, ambiguity $=150^{\circ}$, discontinuity $=50$, faceting $=$ Segment; Right: venus with a cello, ambiguity $=120^{\circ}$, discontinuity $=50$, faceting $=$ Segment. and provides flexibility to choose different discontinuity values for each output (Fig. 11).

Although there is no clear evidence that cubist painters created ambiguity and discontinuity in their paintings with the exact same ideas we used in our system, these two parameters cause similar variety of ambiguity and discontinuity exhibited in the cubist paintings. Accordingly, our user studies (User Study I and User Study III in Appendix A) also support the idea that these parameters vary ambiguity and discontinuity on the outputs.

\subsection{Faceting and artistic style}

Faceting is one of the strongest visual characteristics of analytical cubist paintings. Several observations on the usage of faceting for real cubist paintings are given in Section 3. We have compared four different faceting techniques (Fig. 4), and selected the most efficient approach as the Segment faceting method, as described in Section 4.3.1. Segment faceting technique obeys most of these observations, and therefore reinforces the impression of cubist style for the viewers. User Study II (Appendix A) provides a survey on the comparison of these techniques.

On the other hand, the Segment faceting technique still does not satisfy all properties of analytical cubist style. In real cubist paintings, all facet contours are not boldly defined and fusion of particular facets can be observed. Cubist painters choose some facets to be fused in order to support overall composition. Usage of merely convex quadrilaterals as facet shapes is another problem of our method. Although cubist painters most frequently applied convex quadrilaterals, some painters also used other shapes such as undefined curves, and convex and concave hulls to enhance the borders of the objects.

\subsection{Camera surface}

Our extending view approach enlarges the view by enabling a flexible camera surface. Compared to perspective and orthographic cameras, the proposed camera models increase the number of face counts rendered as indicated in Table 1 . The number of rendered faces is a significant parameter to control the level of ambiguity and the results of User Study III in Appendix A support this claim. This is one of the most important benefits of the proposed camera surfaces. It provides a large surface to select multiple view facets and control the level of ambiguity. Fig. 11, top shows five results of Venus model with different convergence angle values. The leftmost result has $90^{\circ}$ convergence angle, which has a similar result to perspective camera in comparison with the number of face counts rendered. However, the results get more ambiguous as convergence angle is increased from left to right. The correlation of ambiguity with cubist paintings is explained in Sections 3 and 5.1.
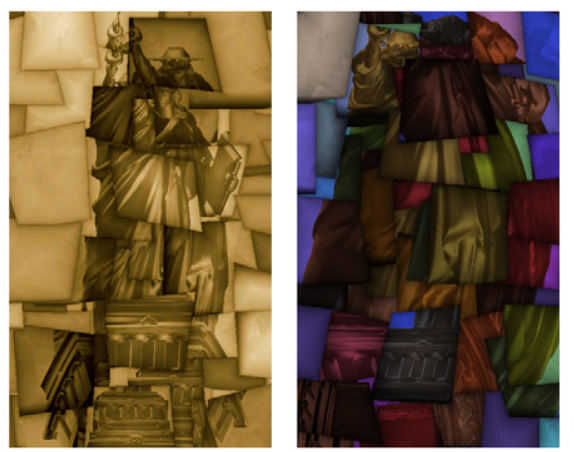

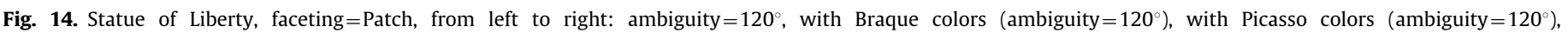
ambiguity $=200^{\circ}$. (For interpretation of the references to color in this figure caption, the reader is referred to the web version of this article.) 
Alternatively, a conventional camera for each facet, similar to Singh's [31] multi-perspective camera model, could be used instead of spherical and cylindrical camera surfaces. As a matter of fact, this model is advantageous to our model in terms of its flexibility for positioning each camera independently. Our current model limits the position of each view facet to given camera surface. However, Singh's method does not give a solution for positioning so many different viewpoints in accordance with cubist paintings in 3D space. It is clear that cubist painters positioned their viewpoints in a spatial relationship not in a random fashion, although their method of building this relationships is not evident. Hence, it is required to have some kind of relationship for the positions of so many viewpoints to create an overall composition and our method provides a simple relationship by fitting each view facet to a flexible camera surface.

A similar problem exists for selection of view direction of each facet independently. It is known that cubist painters aim to show more essential parts of the contents [26]. By using this idea, we have proposed a novel method to adjust view directions automatically as mentioned in Section 4.3.2.

The greater deformation occurring on the output is one of the other disadvantages of our camera surface. On the other hand, this deformation does not completely contradict with the style of analytical cubist paintings. Early analytical cubist paintings, such as Large Nude (Georges Braque, 1908) and Three Women (Pablo Picasso, 1908), exhibit very similar non-linear warps to those that occur in our results, although, in the following periods of analytical cubism (i.e. Braque's Violin and Pitcher, and Picasso's Portrait of Ambroise Vollard), forms have been emphasized with more linear projections. There is actually no common approach of cubist painters for the usage of non-linear or linear effects, when the whole analytical cubism period is considered. Therefore, we provide an option to decrease the greater deformation occurring on the output. This deformation may be avoided spatially by enabling non-deformed perspective view for each facet by changing the term $\alpha$ in Eq. (6), as explained in Section 4.3.2.

\subsection{The opinions of art critics}

We consulted several faculty members of a Fine Arts Department on a few outputs of our model. We asked their opinions on what these outputs capture and what they fail to in the context of analytical cubism. All of them noted that first impression of cubism is strongly achieved. Visual characteristics of cubism stand out with application of multi-perspective rendering and spatial imprecision techniques.

One main critique about the proposed camera model from a scholar was the continuity of the image between some adjacent facets. The complexity of facet relationships is required to be balanced while breaking up overall unity into facets. Although the facets show objects' parts from different view angles, they are not positioned in a random fashion. When we increase the convergence angle of the camera and threshold for the view orientation of the facet, discontinuities between adjacent facets also increase. On the other hand, global position of object parts give the feeling of random placement. For instance, the feet of the female model could appear on the top, near head. We commonly rendered the best results with $120^{\circ}$ to $150^{\circ}$ of convergence angle (ambiguity), and 200 pixels of facet orientation threshold (discontinuity) for the outputs of $1000 \times 1000$ pixels.

Another successful aspect of the outputs indicated by critics was ambiguity created on overall composition. It takes time to recognize the objects like in cubist paintings, but object information is still not lost and overall composition is not vague. Some scholars remarked on the contradiction of pictorial quality within some facets with cubism. Differently than our results, in most of the cubist paintings, the content in a facet is not strongly defined because of painterly style. Abstraction of the content is various on the cubist paintings since each artist has a different brush technique. Therefore, the main focus of this work is applying the common principles present in all analytical cubist paintings rather than brush effects varying for each artist or painting.

Repeated shapes were indicated as a problem for some of the outputs. When we increase the convergence angle of the camera, the amount of repeated shapes increase as orientation of each facet is decided independently than adjacent facets. Decreasing the convergence angle reduces repeated shapes but the feeling of multi-perspective rendering decreases. Our main motivation for not discarding all repeated shapes is that some cubist paintings also having them. For instance, in the Lady with a Guitar (Lyubov Sergeyevna Popova, 1914) there are repeated parts of the guitar as in some of our results.

\subsection{Limitations}

Creating skilled algorithms that can compete with human artists is extremely difficult. Reconstructing an art movement or artistic style imposes limitations on the capturing of style and insight of artists. In order to derive a computational model of an artistic style, analysis of common techniques used on the paintings is the base requirement. However, a common computational analysis method does not exist which necessitates looking for a consensus of art critics on cubist paintings. Although the cubist principles could be clearly described, the painterly style is various and changes from artist to artist. For instance, the contours of facets on our outputs are sharper and regular than cubist paintings of Picasso and Braque. We create facets in a simple relation with the content while cubist artists have created some facets by aligning them with silhouette of the content. In this way, they have used contours in different styles to create certain local harmonies. Other limitations are listed below:

- Our system depends on 3D models to create cubist imagery.

- The proposed camera is limited to Analytical Cubism and the results do not exhibit similarities with other periods of cubism.

- The overall system proposes a common approach to create cubist paintings, which does not allow the user to make local adjustments to the paintings.

- Although Segment faceting method is the preferred one, it takes a long process to create corresponding facets for a scene. A more efficient faceting method is required to create cubist animations.

\section{Conclusion}

We have presented a novel cubist rendering approach for 3D synthetic environments. To this end, the main principles of cubism are applied to generate cubist outputs. The proposed camera model manipulates perspective to expand the view of the scene to be projected. Besides, a perceptual spatial imprecision method is used to break up overall unity into facets while retaining perceptually important parts in the visible areas. Several painterly effects have been performed to enhance visual qualities.

To evaluate the effect of the developed cubist camera on extending the view, we measured the number of visible faces in the rendered images and compared them with the perspective case. The proposed cubist camera model is capable of showing significantly more faces than the traditional perspective camera model. As demonstrated in the user studies, the proposed properties are found to be effective and the final images are highly ranked as similar to analytical cubist paintings by the majority of the subjects.

Although the results are promising, the artistic effects can be further developed by conceiving the brush techniques of Picasso 
and Braque's cubist paintings and integrating them to the current framework.

In addition to the cubist rendering of static computer graphics scenes, we used the proposed algorithm to generate cubist animations in which camera and scene objects are animated while the used facets are static. Developing a more sophisticated cubist animation system, which takes the time dimension into account and animates facets, can be a direction of future work.

\section{Acknowledgments}

This work is supported by the Scientific and Technical Research Council of Turkey (TUBITAK, Project number: 110E029). The authors would like to thank Gaye Çulcuoğlu, Ercan Sağlam, Dominique Tezgör-Kassab, Agnieszka Srokosz, Adam Pekalski and Dilek Kaya from Bilkent University Faculty of Art, Design and Architecture for their invaluable comments and suggestions, and all subjects who participated in the user studies.

\section{Appendix A. User studies}

To further evaluate the success of our study we performed five user studies. In the user studies, we evaluated the efficiency of spatial imprecision with discontinuity parameter, faceting and ambiguity. In addition, we performed two user studies, where we compared our method's results with actual cubist paintings.

To ensure realistic evaluation of parameters side by side, the subjects were required not to have an advanced knowledge of cubism. We performed our user studies with graduate-level computer engineering students. The same 12 subjects ( 4 female, 8 male), whose average age was 24.6, participated in all user studies. We used printed material, which included analytical cubist paintings, synthetic cubist paintings, some other paintings having similar style to cubism, our results, and the outputs of other computer generated cubist results. The content of each card, their parameters and codes referred in user studies are given in Table A1. We applied four different methods of user studies, which are forced choices, matching, card sorting and pile sorting. For forced choices, a number of pairs were given to the subjects, and they were forced to choose one by considering the given question. For the matching study, the subjects were forced to find a match for a given card from the given list. In card sorting and pile sorting, subjects were required to arrange given cards towards given references and task.

User Study I: spatial imprecision and discontinuity. This study evaluated the efficiency and success of spatial imprecision method. There are three parts of this study:

\section{Forced choices}

Number of cards: 12 (A4-A6, A2-A5, A3-A7, A9-A13, A10-A12, A11-A14)

Definition: We showed six pairs of our results to compare random spatial imprecision with saliency-based spatial imprecision technique. Each pair includes one random and one saliency-based result of the same content with the same amount of discontinuity value.

Question: Which image in each pair gives more essential information about the content for each given pair?

Results:

Saliency based spatial imprecision: $76.4 \%$

Random spatial imprecision: $23.6 \%$

Results show that our saliency based method increases the visibility of significant visual information. The goal of the saliency based method is to orient the focus of facets to essential parts of the content. We can also use a random orientation method to create discontinuity. On the other hand, the random method does not guarantee showing essential parts of the objects as the results indicate.

2. Card sorting

Number of cards: 8 (A1-A2-A3-A4, A8-A9-A10-A11)

Definition: We showed two lists of our results to verify the discontinuity variable, which controls spatial imprecision. Each list includes four results of the same content with different discontinuity values.

Task: Sort given two series by considering the discontinuity between patches. The least discontinuous one should be in first order.

Results: 1st list: $\mathrm{A} 1=1.00, \mathrm{~A} 4=2.17, \mathrm{~A} 2=2.83, \mathrm{~A} 3=4.00$

2nd list: $\mathrm{A} 8=1.50, \mathrm{~A} 10=1.67, \mathrm{~A} 9=3.16, \mathrm{~A} 11=3.67$

The results show the average order of each card, and they verify expected sorting. It shows that as we increase the discontinuity variable, the impression of discontinuity was also observed by the viewers.

3. Matching

Number of cards: 6 (A8-A9-A10-A11, E13, E14)

Definition: We showed a list of our results, to compare the degree of discontinuity in our work with real cubist paintings. The list includes four results, two of them with lower discontinuity values and the other two with higher values, to be matched with the given two cubist painting. Base paintings were The Table which has more continuous forms, and Violin and Palette having sharp discontinuities.

Task: Match the given base paintings with one of the paintings in the list, by considering their way of showing the content in terms of discontinuities. You can match both of the two reference paintings with the same painting in the list.

Results:

Lower discontinuity (A8, A10): 83\%, The Table

Higher discontinuity (A9, A11): 75\%, Violin and Palette

As we expected, most of the subjects selected one of results having lower discontinuity to be matched with The Table (E13) and higher discontinuity with Violin and Palette (E14). This shows that the degree of discontinuity in our method correlates with the discontinuity of given cubist paintings.

User Study II: faceting We present four different methods of faceting in our approach. In this user study, we evaluated their similarity to cubist paintings.

\section{Card sorting}

Number of cards: 12 (C1-C2-C3-C4, C5-C6-C7-C8, E1-E14E15-E16)

Definition: We showed two lists of four different outputs with the same content having a different faceting technique. We also provided a list of four cubist paintings as references.

Task: Sort given lists by considering their similarities to reference paintings. First derive an overall style of composition from reference paintings and make your sorting accordingly. Put the most similar one in first order.

Results:

1st list: $\mathrm{C} 3=2.02, \mathrm{C} 1=2.22, \mathrm{C} 2=2.33, \mathrm{C} 4=3.42$

2nd list: $\mathrm{C} 7=2.00, \mathrm{C} 5=2.17, \mathrm{C} 6=2.33, \mathrm{C} 8=3.50$

The results show the average order of each card. Voronoi segmentation, also used in earlier cubist rendering methods, was selected as the most dissimilar one to given cubist paintings. Although our final faceting technique, which satisfies most of faceting rules about cubism we discussed, was sorted in first order, the difference with the other two techniques is not significant. 
Table A1

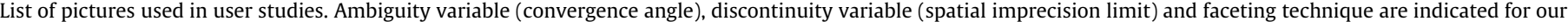
results.

\begin{tabular}{|c|c|c|c|c|}
\hline & Type & Ambiguity & Discontinuity & Faceting \\
\hline A1 & Man, our result & 120 & 0 & Segment \\
\hline $\mathrm{A} 2$ & Man, our result & 120 & 100 & Segment \\
\hline A3 & Man, our result & 120 & 200 & Segment \\
\hline A4 & Man, our result & 120 & 50 & Segment \\
\hline A5 & Man, our result & 120 & Random-100 & Segment \\
\hline A6 & Man, our result & 120 & Random-50 & Segment \\
\hline A7 & Man, our result & 120 & Random-200 & Segment \\
\hline A8 & Venus, our result & 120 & 0 & Segment \\
\hline A9 & Venus, our result & 120 & 100 & Segment \\
\hline $\mathrm{A} 10$ & Venus, our result & 120 & 50 & Segment \\
\hline A11 & Venus, our result & 120 & 200 & Segment \\
\hline A12 & Man, our result & 120 & Random-50 & Segment \\
\hline A13 & Man, our result & 120 & Random-100 & Segment \\
\hline A14 & Man, our result & 120 & Random-200 & Segment \\
\hline $\mathrm{C} 1$ & Man, our result & 120 & 50 & Constant \\
\hline $\mathrm{C} 2$ & Man, our result & 120 & 50 & Patch \\
\hline $\mathrm{C} 3$ & Man, our result & 120 & 50 & Segment \\
\hline $\mathrm{C} 4$ & Man, our result & 120 & 50 & Voronoi \\
\hline C5 & Venus, our result & 120 & 50 & Constant \\
\hline C6 & Venus, our result & 120 & 50 & Patch \\
\hline $\mathrm{C} 7$ & Venus, our result & 120 & 50 & Segment \\
\hline $\mathrm{C} 8$ & Venus, our result & 120 & 50 & Voronoi \\
\hline D1 & Man, our result & 90 & 50 & Segment \\
\hline D2 & Man, our result & 120 & 50 & Segment \\
\hline D3 & Venus, our result & 150 & 50 & Segment \\
\hline D4 & Venus, our result & 180 & 50 & Segment \\
\hline D5 & Venus, our result & 270 & 50 & Segment \\
\hline D6 & Man, our result & 150 & 50 & Segment \\
\hline D7 & Man, our result & 180 & 50 & Segment \\
\hline D8 & Man, our result & 270 & 50 & Segment \\
\hline D9 & Venus, our result & 90 & 50 & Segment \\
\hline D10 & Venus, our result & 120 & 50 & Segment \\
\hline E1 & $\begin{array}{l}\text { Girl with a Mandolin } \\
\text { (Pablo Picasso, 1910) }\end{array}$ & & & \\
\hline E2 & $\begin{array}{l}\text { Violing and Pitcher } \\
\text { (Georges Braque, 1910) }\end{array}$ & & & \\
\hline E3 & $\begin{array}{l}\text { Wilhelm Uhde } \\
\text { (Pablo Picasso, 1910) }\end{array}$ & Low & & \\
\hline E4 & $\begin{array}{l}\text { The Portuguese } \\
\text { (Georges Braque, 1911) }\end{array}$ & & & \\
\hline E5 & $\begin{array}{l}\text { Fruit and Bowl } \\
\text { (Pablo Picasso) }\end{array}$ & & & \\
\hline E6 & $\begin{array}{l}\text { Mandolin and Guitar } \\
\text { (Pablo Picasso, 1924) }\end{array}$ & & & \\
\hline E7 & $\begin{array}{l}\text { Composition VII } \\
\text { (Kandinsky, 1913) }\end{array}$ & & & \\
\hline E8 & $\begin{array}{l}\text { Bibemus Quarry } \\
\text { (Cezanne, 1895) }\end{array}$ & & & \\
\hline E9 & Our result & & & Segment \\
\hline E10 & Our result & & & Segment \\
\hline E11 & Collomosse and Hall & & & Voronoi \\
\hline E12 & Klein et al. & & & \\
\hline E13 & $\begin{array}{l}\text { The Table } \\
\text { (Georges Braque, 1910) }\end{array}$ & & Low & \\
\hline E14 & $\begin{array}{l}\text { Violin and Palette } \\
\text { (Georges Braque, 1909) }\end{array}$ & & High & \\
\hline E15 & $\begin{array}{l}\text { Nude } \\
\text { (Pablo Picasso, 1910) }\end{array}$ & & & \\
\hline E16 & $\begin{array}{l}\text { Guitar Player } \\
\text { (Pablo Picasso, 1910) }\end{array}$ & High & & \\
\hline
\end{tabular}
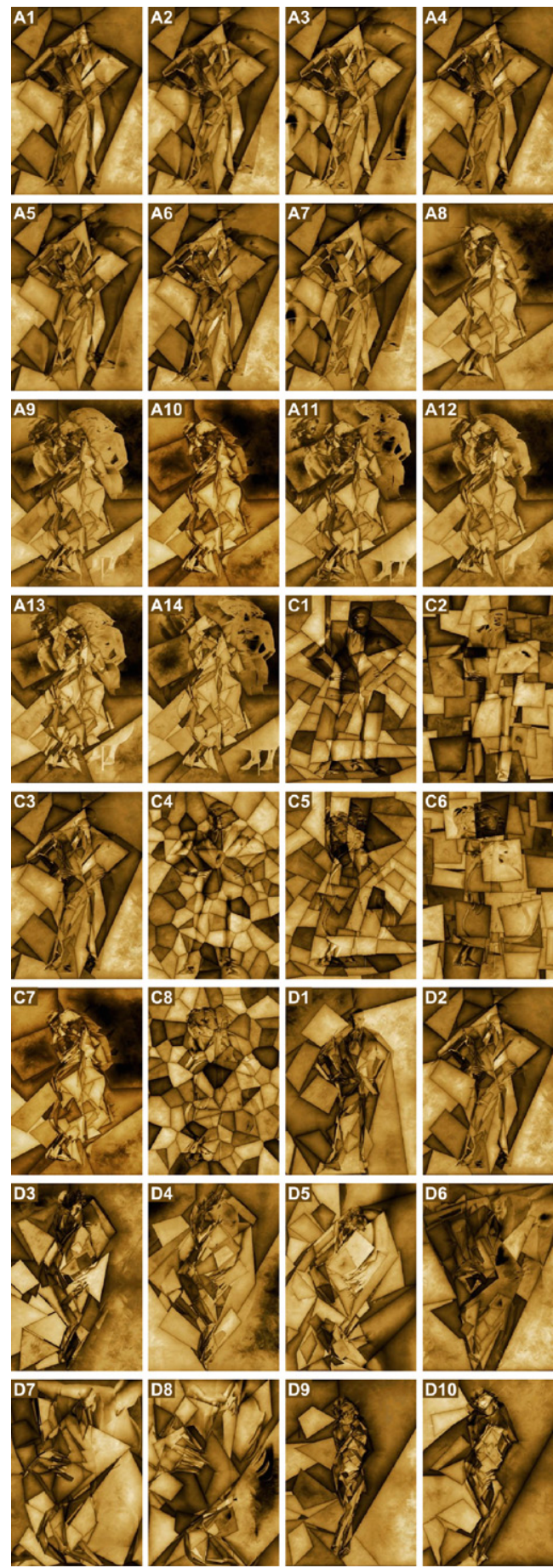

User Study III: ambiguity.

\section{Card sorting}

Number of cards: 10 (D1-D2-D6-D7-D8, D3-D4-D5-D9-D10) Definition: We showed two lists of our results to verify the ambiguity variable, which increases the camera surface. Each list includes four results of the same content with different ambiguity values.
Task: Sort given two series by considering overall ambiguity of paintings. The least ambiguous one should be in first order. Results: 1 st list: $\mathrm{D} 1=1.33, \mathrm{D} 2=2.17, \mathrm{D} 7=3.00, \mathrm{D} 6=3.83$, D8 $=4.67$

2nd list: D9 $=1.67, \mathrm{D} 10=2.42, \mathrm{D} 3=2.83, \mathrm{D} 4=3.75, \mathrm{D} 5=4.33$

The results show the average order of each card. The study results shows that as we increase the ambiguity variable, which means increasing camera surface in our 
Table A2

Correlation table of given cards in User Study V.

\begin{tabular}{|c|c|c|c|c|c|c|c|c|c|c|c|c|c|c|c|c|}
\hline & E1 & E2 & E3 & E4 & E5 & E6 & E7 & E8 & E9 & E10 & E11 & E12 & E13 & E14 & E15 & E16 \\
\hline E1 & 12 & & & & & & & & & & & & & & & \\
\hline E2 & 5 & 12 & & & & & & & & & & & & & & \\
\hline E3 & 9 & 4 & 12 & & & & & & & & & & & & & \\
\hline E4 & 1 & 1 & 3 & 12 & & & & & & & & & & & & \\
\hline E5 & 1 & 0 & 1 & 2 & 12 & & & & & & & & & & & \\
\hline E6 & 0 & 0 & 1 & 1 & 7 & 12 & & & & & & & & & & \\
\hline E7 & 0 & 1 & 1 & 5 & 3 & 3 & 12 & & & & & & & & & \\
\hline E8 & 0 & 2 & 1 & 4 & 4 & 3 & 9 & 12 & & & & & & & & \\
\hline E9 & 7 & 3 & 7 & 0 & 2 & 2 & 0 & 0 & 12 & & & & & & & \\
\hline E10 & 2 & 2 & 2 & 2 & 2 & 3 & 0 & 0 & 5 & 12 & & & & & & \\
\hline E11 & 0 & 2 & 1 & 1 & 6 & 6 & 3 & 3 & 1 & 1 & 12 & & & & & \\
\hline E12 & 1 & 2 & 0 & 3 & 5 & 3 & 1 & 0 & 1 & 3 & 6 & 12 & & & & \\
\hline E13 & 2 & 4 & 0 & 1 & 0 & 2 & 0 & 1 & 1 & 3 & 0 & 2 & 12 & & & \\
\hline E14 & 4 & 2 & 5 & 2 & 0 & 3 & 1 & 1 & 3 & 1 & 1 & 1 & 5 & 12 & & \\
\hline E15 & 0 & 4 & 0 & 3 & 0 & 0 & 3 & 4 & 1 & 3 & 1 & 3 & 5 & 2 & 12 & \\
\hline E16 & 1 & 1 & 1 & 4 & 0 & 2 & 3 & 1 & 0 & 4 & 1 & 2 & 4 & 2 & 4 & 12 \\
\hline
\end{tabular}

model, the impression of ambiguity was also observed by the viewers.

2. Matching

Number of cards: 6 (D4-D5-D9-D10, E16, E3)

Definition: We showed a list of our results to compare the degree of ambiguity in our work with real cubist paintings. The list includes four results, two of them with lower ambiguity values and other two with higher values, to be matched with given two cubist paintings. Reference paintings were Guitar Player (E16), which has more ambiguous forms, and Wilhelm Uhde (E3), having more legible forms.

Task: Match given reference paintings with either one of the paintings in the list by considering their way of showing the content in terms of ambiguity. You can match both of two reference paintings with the same painting in the list.

Results: Lower ambiguity (D4, D5): 91\%, Wilhelm Uhde Higher ambiguity (D9, D10): 75\%, Guitar Player

As we expected, most of the viewers chose one of the results having higher ambiguity to be matched with Guitar Player and lower ones with Wilhelm Uhde. Thus, the amount of ambiguity in our model has a correspondence with the given cubist paintings.

User Study IV: ranking.

1. Card sorting

Number of cards: 12 (E1, E2, E3-E4-E5-E6-E7-E8-E9-E10E11-E12)

Definition: We showed a list of ten paintings, which includes two real analytical cubist paintings (E3, E4), two synthetic cubist paintings $(E 5,56)$, one painting of Kandinsky (E7), one painting of Cezanne (E8), two outputs of our model (E9, E10), one output of Collomosse and Hall's method [18] (E11) and one output of Klein et al.'s method [21] (E12). We also gave two analytical cubist paintings (E1, E2) as references for the task. The goal was to compare the performance of our method with real cubist paintings and other methods in terms of their similarity to cubist style. We used grayscale versions of all outputs to prevent color-based biases.

Task: Sort the given list by considering their similarity in terms of pictorial style to given reference paintings. The most similar one should be in first order.

Results:

List: $\mathrm{E} 3=1.92, \quad \mathrm{E} 9=2.75, \quad \mathrm{E} 4=3.58, \mathrm{E} 10=3.67, \quad \mathrm{E} 11=6.33$, $\mathrm{E} 5=7.0, \mathrm{E} 7=7.0, \mathrm{E} 8=7.16, \mathrm{E} 6=7.67, \mathrm{E} 12=7.92$

Results show the average order of each card. E3, E9, E4 and E10 have a significant ranking difference with other cards. These are two analytical cubist paintings and our results. Our method, compared with other cubist rendering methods, demonstrated a better ranking. Although Collomosse and Hall's method satisfies most of the cubist properties, the faceting technique used in our algorithm provides a better application of cubist principles than the Voronoi method used in that technique. This ranking also shows that synthetic cubist paintings have a sharp difference of style compared to analytical cubism as we asserted. We used Kandinsky's painting, since his paintings have similar shape structures with analytical cubism but they do not have any view-independent projection principle. On the other hand, Cezanne's painting has similar concepts with analytical cubism but its pictorial style is significantly different. As a matter of fact, neither of the paintings of Kandinsky and Cezanne were found similar to analytical cubist paintings in the results.

User Study V: similarities.

\section{Pile sorting}

Number of cards: 16 (E1...E16)

Definition: We showed a list of cards to make another comparison with other outputs and paintings. The list includes the same cards of User Study IV with four additional analytical cubist paintings.

Task: Form four groups of sixteen cards. Each group should have four cards. Consider similarities of overall approach and composition of paintings, and not color and the content.

Results:

Correlation table (Table A2) shows the number of occurrences (N.o.O) of each card with other cards in the same group. Results of this study verify results of User Study IV. For instance, N.o.O of E1, which is an analytical cubist painting, with other analytical cubist paintings is 22 . N.o.O of E9, our result, with other analytical cubist painting is 24 . This value is 7 for E11, Collomosse and Hall's method, 11 for E12, Klein's method, 4 for E5, a synthetic cubist painting of Picasso. Average N.o.O of an analytical cubist painting with other analytical cubist paintings is 19 , and it is 21.5 for our results. This shows that our results have similar values with real analytical cubist paintings.

\section{References}

[1] Gooch B, Gooch A. Non-photorealistic rendering. AK Peters Ltd; 2001.

[2] Zeng K, Zhao M, Xiong C, Zhu SC. From image parsing to painterly rendering. ACM Trans Graph 2009;29(2):1-11. 
[3] Xu X, Zhang L, Wong TT. Structure-based ascii art. In: ACM SIGGRAPH 2010 papers. SIGGRAPH '10. New York, NY, USA: ACM; 2010. p. 52:1-10.

[4] Arnheim R. Art and visual perception. University of California Press; 1954 p. 50-60.

[5] Seitz SM, Kim J. Multiperspective imaging. IEEE Comput Graph Appl 2003;23:16-9.

[6] Glassner A. Digital cubism. IEEE Comput Graph Appl 2004;24:82-90.

[7] Glassner A. Digital cubism, part 2. IEEE Comput Graph Appl 2004;24: 84-95.

[8] Löffelmann H, Gröller ME. Ray tracing with extended cameras. J Visual Comput Animation 1996;7(4):211-27.

[9] Yu J, McMillan L. General linear cameras. In: ECCV (2); 2004. p. 14-27.

[10] Yu J, McMillan L, A framework for multiperspective rendering. In: Eurographics symposium on rendering. Norrkoping, Sweden; 2004

[11] Popescu V, Rosen P, Adamo-Villani N. The graph camera. In: ACM SIGGRAPH Asia 2009 papers. SIGGRAPH Asia '09. New York, NY, USA: ACM; 2009. p. 158:1-8.

[12] Taguchi Y, Agrawal A, Veeraraghavan A, Ramalingam S, Raskar R. Axialcones: modeling spherical catadioptric cameras for wide-angle light field rendering. In: ACM SIGGRAPH Asia 2010 papers. SIGGRAPH ASIA '10. New York, NY, USA: ACM; 2010. p. 172:1-8.

[13] Brosz J, Samavati FF, Sheelagh MTC, Sousa MC. Single camera flexible projection. In: Proceedings of the 5th international symposium on nonphotorealistic animation and rendering. NPAR '07. New York, NY, USA: ACM; 2007. p. 33-42.

[14] Wood DN, Finkelstein A, Hughes JF, Thayer CE, Salesin DH. Multiperspective panoramas for cel animation. In: Proceedings of the 24th annual conference on computer graphics and interactive techniques. SIGGRAPH '97. New York, NY, USA: ACM Press/Addison-Wesley Publishing Co.; 1997. p. 243-50.

[15] Rademacher P, Bishop G. Multiple-center-of-projection images. In: Proceedings of the 25th annual conference on computer graphics and interactive techniques. SIGGRAPH '98. New York, NY, USA: ACM; 1998. p. 199-206.

[16] Agrawala M, Zorin D, Munzner T. Artistic multiprojection rendering. In: Proceedings of the eurographics workshop on rendering techniques 2000. London, UK: Springer-Verlag; 2000. p. 125-36.
[17] Coleman P, Singh K. Ryan: rendering your animation nonlinearly projected. In: Proceedings of the 3rd international symposium on non-photorealistic animation and rendering. NPAR '04. New York, NY, USA: ACM; 2004. p. 129-56.

[18] Collomosse JP, Hall PM. Cubist style rendering from photographs. IEEE Trans Visual Comput Graph 2002;4:443-53.

[19] Agarwala A, Agrawala M, Cohen M, Salesin D, Szeliski R. Photographing long scenes with multi-viewpoint panoramas. In: ACM SIGGRAPH 2006 papers. SIGGRAPH '06. New York, NY, USA: ACM; 2006. p. 853-61.

[20] Klein AW, pike J, Sloan P, Colburn RA, Finkelstein A, Cohen MF. Video cubism. Technical report; Microsoft Research; 2001.

[21] Klein AW, Sloan PPJ, Finkelstein A, Cohen MF. Stylized video cubes. In Proceedings of the 2002 ACM SIGGRAPH/eurographics symposium on computer animation. SCA '02. New York, NY, USA: ACM; 2002. p. 15-22.

[22] Song YZ, Rosin PL, Hall PM, Collomosse JP. Arty shapes. In: Computational aesthetics; 2008. p. 65-72.

[23] Rubin W. Picasso and braque: pioneering cubism. The Museum of Modern Art, New York; 1989. p. 15-63.

[24] Poggi C. In defiance of painting: cubism, futurism, and the invention of collage. Yale University Press; 1992. p. 31-43.

[25] Livingstone M. Vision and art: the biology of seeing. ABRAMS; 2002 p. 101-5.

[26] Cooper D. The cubist epoch. Phaidon Press; 1994 p. 30-60.

[27] Cour T, Benezit F, Shi J. Spectral segmentation with multiscale graph decomposition. In: Proceedings of the 2005 IEEE computer society conference on computer vision and pattern recognition (CVPR'05) - Volume 2 Volume 02. CVPR '05. Washington, DC, USA: IEEE Computer Society. ISBN 0-7695-2372-2; 2005. p. 1124-31.

[28] Itti L, Koch C. Computational modeling of visual attention. Nat Rev Neurosci 2001;2(3):194-203.

[29] Lee CH, Varshney A, Jacobs DW. Mesh saliency. In: ACM SIGGRAPH 2005 papers. SIGGRAPH '05. New York, NY, USA: ACM; 2005. p. 659-66.

[30] Hertzmann A. Non-photorealistic rendering and the science of art. In: Proceedings of the 8th international symposium on non-photorealistic animation and rendering. NPAR '10. New York, NY, USA: ACM. ISBN 978-14503-0125-1; 2010. p. 147-57. http://dx.doi.org/10.1145/1809939.1809957, $\langle$ http://doi.acm.org/10.1145/1809939.1809957〉.

[31] Singh K. A fresh perspective. In: Graphics interface; 2002. p. 17-24. 